\title{
Actitud estudiantil ante el plagio y la conciencia para evitarlo
}

\section{Students' attitudes towards plagiarism and awareness to avoid it}

DOI: https://doi.org/10.32870/dse.v0i23.730

\author{
Edrei del Carmen Izquierdo Alejandro* \\ Deneb Elí Magaña Medina** \\ Román Alberto Quijano García***
}

\begin{abstract}
Resumen
El objetivo de esta investigación fue determinar las actitudes hacia el plagio de los estudiantes de contabilidad de una universidad pública del sureste de México, y los principales factores que lo predicen. La población del estudio se conformó por una muestra no probabilística representativa de 200 estudiantes de contaduría pública. Se desarrolló un cuestionario denominado Percepción del estudiante universitario sobre el plagio (PEUP), que fue validado por expertos, y presenta valores aceptables de fiabilidad $(a \geq$ .70). Su estructura teórica fue confirmada a través del análisis factorial exploratorio y confirmatorio. Los resultados indican que $10.5 \%$ de los estudiantes aceptan no dar crédito a los autores originales cuando realizan sus asignaciones académicas, pero $41 \%$ de la población encuestada reconoce tener conciencia sobre la importancia del plagio y cómo evitarlo. Los resultados de la regresión lineal jerárquica señalan que la conciencia para evitar el plagio es el factor que explica la mayor parte de las actitudes personales hacia el mismo $(\beta=.39, p=.000)$, seguido de las competencias para evitarlo $(\beta=.33, p=.000)$; y por último, las condiciones percibidas por el estudiante que le permiten realizarlo $(\beta=.27, p=.000)$. Se concluye que la conciencia del estudiante sobre el plagio es el principal predictor del mismo, lo cual reafirma la premisa de que el plagio es un asunto de ética y valores, tanto individuales como colectivos.
\end{abstract}

Palabras clave: Plagio académico - ética - educación superior.

\section{Abstract}

Our research aimed to determine the attitudes of students of a major in Accounting at a public university in southeastern Mexico towards plagiarism, and the main factors that predict it. Our representative non-probability sample comprised 200 students of Public Accounting. A questionnaire on University

* Licenciada en Contaduría. Universidad Juárez Autónoma de Tabasco. Colaboradora en los proyectos de investigación: “Diagnóstico sobre el interés, condiciones y limitantes de los estudiantes de pregrado y posgrado en las disciplinas económico administrativas para realizar investigación o desarrollo tecnológico en el sureste de México (Proyecto de RED-PRODEP)" y "Factores asociados al logro académico de estudiantes de telesecundaria en el estado de Tabasco", Proyecto INEE-CONACYT. Tabasco, México. edrei.izquierdo96@gmail.com

** Doctora en Ciencias Administrativas: Gestión Socioeconómica. SNI I. División Académica de Ciencias Económico Administrativas de la Universidad Juárez Autónoma de Tabasco. México. deneb_72@yahoo.com

***Doctor en Gestión Estratégica y Políticas de Desarrollo. Profesor investigador, líder del cuerpo académico de Innovación en las organizaciones. Adscrito al área de Finanzas. Facultad de Contaduría y Administración de la Universidad Autónoma de Campeche. México. rq6715@ hotmail.com 
Students' Perception of Plagiarism (USPP) was developed. It was validated by experts and shows acceptable reliability values $(a \geq .70)$. Its theoretical structure was further confirmed through exploratory and confirmatory factor analysis. Our results indicate that $10.5 \%$ of the students admitted not giving credit to the original authors when they make their academic assignments, but $41 \%$ of the surveyed population acknowledge being aware of the importance of knowing what plagiarism is and how to avoid it. The results of the hierarchical linear regression indicate that awareness to avoid plagiarism is the factor that explains most of the personal attitudes towards it $(\beta=.39, p=.000)$, followed by the skills to avoid it $(\beta=.33, p=.000)$ and finally the conditions perceived by the students that allow them to do it $(\beta=$ $.27, \mathrm{p}=.000)$. We concluded that the student's awareness of plagiarism is its main predictor, which reinforces the premise that plagiarism is a matter of ethics and values, both individual and collective.

Key words: Academic plagiarism - ethics - higher education.

\section{Introducción}

El plagio es un problema multifacético, en especial cuando se refiere a los alumnos, pues no hay una línea clara entre los factores personales que lo provocan, la facilidad con que se puede realizar, la ausencia de competencias producto de pobres estrategias didácticas o de la indiferencia de las autoridades educativas para evitarlo, ya sea a través de estrategias de prevención o sanciones.

Culwin y Lancaster (2001) señalan que los alumnos presentan conductas poco éticas como el plagio, porque saben que todos lo hacen y, por ende, se crea una norma. Esta situación, como lo señalan Schrimsher, Northrup y Alverson (2011), provoca que los alumnos no vean el plagio como algo incorrecto. Pero al igual que diversos autores sobre el tema (Gantús, 2016; Inclán, 2016; Jones, 2011; Miranda, 2013; Yankelevich, 2016), se coincide en señalar que esta conducta refiere un importante problema de valores éticos individuales y colectivos, tanto de alumnos como de profesores y autoridades educativas.

Diversas investigaciones en distintos países señalan que los alumnos admiten que con frecuencia copian texto sin citas, siendo el internet la principal fuente de consulta (Bautista, Sánchez, Canto, 2017; Chu, Hu, Ng, 2019; Culwin, Lancaster, 2001; Gómez, Francisco, Moreno, 2016; Maurer, Kappe, 2006.

Morales (2016) señala con acierto que la actual Ley Federal del Derecho de Autor en México no contempla ninguna definición de plagio ni una referencia a este concepto. Esta situación brinda las condiciones necesarias para obviar el problema del plagio como una conducta que debe ser sancionada y sobre la que se hace complicada una legislación (Hannabuss, 2001).

El objetivo del estudio fue determinar las actitudes de los estudiantes de contabilidad de una universidad pública del sureste de México, y los principales factores que lo predicen, tales como la conciencia y competencias necesarias para evitar el plagio y las facilidades que se perciben para realizarlo.

Diáloqos sobre Educación año 12 | número 23 | julio-diciembre 2021 | ISSN 2007-2171 
Al igual que Smith, Ghazali y Noor (2007), se decidió abordar una población de estudiantes de contaduría, en primer término, por ser una disciplina tradicionalmente catalogada como profesionalizante, y porque en muchos casos se subestiman las actividades de investigación. Por otro lado, la profesión percibe la honestidad e integridad de sus miembros como características importantes para conservar la confianza del público en sus actividades de contabilidad, auditoría y asesoría, reguladas con un código de ética específico para la profesión.

Por otra parte, se concuerda con Comas, Sureda y Trobat (2011) en que existen pocos aportes empíricos que se centren en cómo los estudiantes universitarios perciben el plagio desde la perspectiva personal y de cada contexto en particular.

Diversos autores (Díaz, Brito, Nieto, Muñoz, 2019; Hosny, Shameem, 2014; Hu, Sun, 2016) han realizado importantes aportaciones empíricas que presentan los principales factores estudiados sobre las actitudes del estudiante universitario ante esta problemática, brindando estadísticas poco alentadoras sobre su percepción. Sin embargo, en México son escasos los trabajos que aborden la problemática desde la mirada estudiantil y no se encontró un referente empírico para las variables y población en estudio.

\section{Revisión de literatura}

El primer reto que supone esta problemática está en la definición del concepto plagio. Se coincide con Maurer, Kappe y Zaka (2006) cuando afirman que no existe una definición válida de plagio textual que no dependa del dominio, lo que hace más difícil esta labor.

Diversos autores (Bautista et al., 2017; Berlinck, 2011; Hernández, 2016; Merino, Charter, 2010; Miranda, 2013) presentan su definición iniciando con la etimología de la palabra que se origina del latín, esencialmente del concepto de secuestro, o del griego, como trapacero o falso.

Este origen genera definiciones (Díaz et al., 2019; Hannabuss, 2001; Kattan et al., 2017; López, 2014; Maurer et al., 2006; Meuschke, Gipp, 2013) que tienen en común tres elementos: a) la copia de palabras sin crédito al autor original, b) el presentar ideas de otros autores como propias, y c) la intencionalidad de estos actos para beneficio propio.

De estas definiciones, se puede diferenciar la que presentan Culwin y Lancaster (2001: 36) para el plagio estudiantil, descrita como: "plagio con la intención de obtener crédito académico".

Cardoso (2016: 188) hace hincapié en un tipo de plagio más profesional, al que refiere como el plagio académico y que se practica en las organizaciones educativas, "consiste en la apropiación indebida de la producción de conocimiento técnico y científico".

Por último, están las definiciones que se sustentan en la titularidad de los derechos de autor (Enders, Hoover, 2006; Meuschke, Gipp, 2013; Miranda, 2013; Rohwer, Wager, Young, Garner, 2018; Timal, Sánchez, 2017), que discuten sobre la propiedad de las palabras e ideas, del consentimiento de los autores y de los beneficios que se otorgan o violan al realizar un plagio. Es necesario remarcar que la legislación de cada país es un elemento a considerar cuando se discute sobre derechos de autor. 
En relación con los tipos de plagio, también se pueden encontrar diversas clasificaciones, Maurer et al. (2006: 1051) refieren en primer término una clasificación en relación con el nivel de conciencia que el individuo posee sobre el plagio, catalogándolo como: a) accidental: aquel que se comete por falta de conocimiento sobre lo que es el plagio o la carencia de comprensión de los esquemas de citado y referenciado, $b$ ) no intencional: aquel que se da por la gran cantidad de información disponible que influye en los pensamientos e ideas que pueden surgir, $c$ ) intencional: referido al acto deliberado de copiar en forma completa o en parte el trabajo de otra persona sin dar crédito al autor original, y d) autoplagio: que supone el empleo del trabajo propio publicado sin referirse al original.

Otras clasificaciones se concentran en la forma y cantidad de información que se utiliza de otros autores (Bhutto, Mamdani, 2019; Cardoso, 2016) o en la manera en que se realiza el plagio (Comas et al., 2011), si es a través de internet o de la copia de fuentes impresas. Meuschke y Gipp (2013: 51) introducen un tipo de plagio particularmente empleado por los estudiantes, al que denominan disfraz técnico, definido como "las técnicas que explotan las debilidades de los métodos de detección actuales para hacer que el contenido plagiado no sea reconocido por algún programa informático", esto incluye la traducción a un idioma diferente (Morató, 2012) o el empleo de sinónimos (Timal, Sánchez, 2017).

El plagio también es concebido no solo en la forma en que se realiza sino también como un fraude en la suplantación de los créditos del autor, conocido como escritor fantasma (Enders, Hoover, 2006; Morató, 2012; Soto, 2012; Vera, 2016), denominado de dicha forma pues el autor recibe un pago por la suplantación de la identidad en la autoría del trabajo que se presenta.

Mas allá del tipo de plagio, esta investigación se centra en los factores que lo generan y, desde la perspectiva del grupo de investigación, las causas del plagio pueden agruparse en cuatro grandes categorías: a) las causas personales, atribuidas esencialmente a sus actitudes, personalidad o carencias del estudiante; $b$ ) las que se atribuyen a las deficiencias del proceso de enseñanza aprendizaje, las cuales son referidas en su mayoría a las carencias que los profesores y programas educativos tienen sobre el tema; c) las que se atribuyen a las deficiencias en la regulación por parte de las organizaciones educativas, referidas a la ausencia, tanto en su definición como en la prevención, y en los esquemas de sanción; y por último, $d$ ) aquellas que se pueden atribuir al contexto en general, es decir, aquellas condiciones que han facilitado o promovido que el estudiante realice plagio (Bautista et al., 2017; Chu et al., 2019; Díaz et al., 2019; Gómez et al., 2016; Inclán, 2016; Jones, Reid, Bartlett, 2006; Morató, 2012; Malcolm et al., 2007; Soto, 2012; Zimerman, 2012) (Tabla 1). 
Tabla 1. Causas atribuidas al plagio estudiantil

\begin{tabular}{|c|c|c|c|}
\hline Causas personales & $\begin{array}{l}\text { Deficiencias del } \\
\text { proceso enseñanza } \\
\text { aprendizaje }\end{array}$ & $\begin{array}{l}\text { Deficiencias } \\
\text { regulatorias }\end{array}$ & Causas contextuales \\
\hline $\begin{array}{l}\text { - Falta de motivación. } \\
\text { - Procrastinación. } \\
\text { - Pereza. } \\
\text { - Percepción de pérdida } \\
\text { de tiempo. } \\
\text { - Ineficiente administra- } \\
\text { ción del tiempo. } \\
\text { - No sabe citar. } \\
\text { - Barrera del idioma. } \\
\text { - Falta de confianza en } \\
\text { sus capacidades de } \\
\text { redacción. } \\
\text { - No entiende lo que es } \\
\text { el plagio. } \\
\text { - Convencimiento de } \\
\text { que no será descubier- } \\
\text { to. } \\
\text { - Falta de ética. }\end{array}$ & $\begin{array}{l}\text { - Sobrecarga de labores } \\
\text { académicas. } \\
\text { - Indiferencia de los } \\
\text { profesores. } \\
\text { - Ausencia de asesoría o } \\
\text { retroalimentación. } \\
\text { - Ausencia del tema en } \\
\text { los programas } \\
\text { académicos. } \\
\text { - Ausencia de las com- } \\
\text { petencias de redacción } \\
\text { requeridas en el profe- } \\
\text { sorado. }\end{array}$ & $\begin{array}{l}\text { - Políticas y sanciones } \\
\text { poco claras o inexisten- } \\
\text { tes sobre el plagio aca- } \\
\text { démico. } \\
\text { - Poca o nula infraes- } \\
\text { tructura informática } \\
\text { para su detección } \\
\text { (software detector de } \\
\text { plagio). } \\
\text { - Ausencia de una de- } \\
\text { finición operacional de } \\
\text { plagio en los códigos de } \\
\text { ética }\end{array}$ & $\begin{array}{l}\text { - Estrategias didácticas } \\
\text { poco eficientes. } \\
\text { - Facilidades que brinda } \\
\text { el internet para copiar y } \\
\text { pegar. } \\
\text { - Desarrollo en las tec- } \\
\text { nologías de la informa- } \\
\text { ción y la comunicación. } \\
\text { - Presión social del } \\
\text { grupo. } \\
\text { - Falta de una cultura } \\
\text { que valore el esfuerzo } \\
\text { del trabajo académico. }\end{array}$ \\
\hline
\end{tabular}

\section{Método}

\section{Participantes}

La población en estudio se conformó por una muestra no probabilística representativa, 200 alumnos de 528, del programa de contaduría pública de una universidad pública del sureste de México (UJAT, 2019), que cumplían los criterios de inclusión de la muestra. Se tomó como referente que los alumnos no fueran de nuevo ingreso, considerando únicamente a los alumnos de tercer ciclo (segundo año) en adelante, pues la finalidad era incluir en la muestra alumnos que hubieran tenido al menos un año en la universidad.

\section{Procedimiento}

Se solicitó a los participantes que contestaran el cuestionario de manera autoaplicada, a papel y lápiz y con acompañamiento de uno de los miembros del equipo de investigación. La aplicación se llevó a cabo durante los meses de octubre a diciembre de 2019.

\section{Diseño del instrumento}

A partir de la revisión documental de los instrumentos se desarrolló un cuestionario basado esencialmente en el trabajo de Smith et al. (2007), pero se consideraron para su traducción y 
adaptación las aportaciones de Hosny y Shameem (2014), Cebrián, Raposo, Cebrián y Sarmiento (2018), López (2014) y Schrimsher, Northrup y Alverson (2011). Es necesario señalar que, si bien el instrumento original fue validado por sus autores, la versión empleada fue una traducción y adaptación aplicada a un contexto totalmente diferente, como lo es México, lo cual también representa una de las aportaciones del estudio.

La validez de contenido se realizó de manera cualitativa, con la aportación del juicio de cinco expertos, entre ellos, profesores, estudiantes de posgrado y autoridades académicas con experiencia en el tema. Se les pidió revisar el cuestionario y que brindaran una retroalimentación sobre la redacción y traducción de este para cada ítem.

El instrumento quedó conformado por cuatro medidas y tres variables que estiman la percepción del estudiante universitario sobre el plagio (PEUP). Las variables que lo conforman son: actitudes personales hacia el plagio (APAP), condiciones para evitar el plagio (CPP), que está conformada por dos factores: conciencia para evitar el plagio (COEP) y competencia para evitar el plagio (CMEP), y facilidad percibida para plagiar (FPPP) (tabla 2).

El instrumento se diseñó en una escala tipo Likert con cinco opciones de respuesta: $1=$ completamente en desacuerdo, 2 =en desacuerdo, 3 = ni de acuerdo ni en desacuerdo, $4=$ de acuerdo y $5=$ completamente de acuerdo. Adicionalmente, se agregaron elementos demográficos generales como el sexo y la edad, y variables del contexto como el ciclo escolar y el promedio. Se incluyó un apartado general para indicar cuáles eran las principales fuentes de consulta, cuáles serían las consecuencias que debería afrontar un estudiante que comete plagio, y cómo daba crédito a los autores en sus tareas escolares. 
Tabla 2. Tabla de especificaciones de los factores que comprenden el constructo "Percepción del Estudiante Universitario sobre el Plagio (PEUP)"

\begin{tabular}{|c|c|c|c|}
\hline Variable & Factores & Definición Operacional & Ítems \\
\hline \multicolumn{2}{|c|}{$\begin{array}{l}\text { Actitudes Personales } \\
\text { Hacia el Plagio (APHP). }\end{array}$} & $\begin{array}{l}\text { En esta variable identifica } \\
\text { las actitudes personales } \\
\text { de los estudiantes en rela- } \\
\text { ción con las actividades de } \\
\text { plagio. }\end{array}$ & $\begin{array}{l}\text { No tengo ganas de trabajar ni aprender a evitar el plagio. } \\
\text { No veo la necesidad de tener conocimiento sobre el plagio } \\
\text { para mi futuro. } \\
\text { Quiero evitar el trabajo duro y por eso mejor copio de } \\
\text { internet. } \\
\text { No me interesa el tema de plagio. } \\
\text { Cometo plagio porque todos lo hacen. }\end{array}$ \\
\hline \multirow[t]{2}{*}{$\begin{array}{l}\text { Condicio- } \\
\text { nes Para } \\
\text { Evitar el } \\
\text { Plagio } \\
\text { (CPEP) }\end{array}$} & $\begin{array}{l}\text { Concien- } \\
\text { cia para } \\
\text { evitar el } \\
\text { plagio } \\
\text { (COEP) }\end{array}$ & $\begin{array}{l}\text { En este factor se indaga } \\
\text { sobre el nivel de concien- } \\
\text { cia al momento de come- } \\
\text { ter el plagio, de saber si } \\
\text { razonan antes de come- } \\
\text { terlo o lo ven como una } \\
\text { acción normal. }\end{array}$ & $\begin{array}{l}\text { No entiendo lo que es plagio. } \\
\text { No veo el plagio como un problema. } \\
\text { Siento que no es importante reconocer al escritor original. }\end{array}$ \\
\hline & $\begin{array}{l}\text { Compe- } \\
\text { tencia } \\
\text { para } \\
\text { evitar el } \\
\text { plagio } \\
\text { (CMEP) }\end{array}$ & $\begin{array}{l}\text { En esta factor se identifica } \\
\text { la perspectiva que tiene de } \\
\text { sí mismo el alumno en re- } \\
\text { lación con las habilidades } \\
\text { académicas que le permi- } \\
\text { tirían evitar el plagio. }\end{array}$ & $\begin{array}{l}\text { No tengo la confianza para preparar una buena tarea. } \\
\text { Me resulta difícil construir oraciones. } \\
\text { Tengo pocas habilidades de investigación. } \\
\text { Tengo dificultad para entender artículos en inglés. } \\
\text { No sé cómo reconocer adecuadamente el autor a través } \\
\text { de citas. } \\
\text { No sé citar correctamente. }\end{array}$ \\
\hline \multicolumn{2}{|c|}{$\begin{array}{l}\text { Facilidad para plagiar } \\
\text { (FPPP) }\end{array}$} & $\begin{array}{l}\text { Percepción del estudiante } \\
\text { sobre la facilidad de co- } \\
\text { meter plagio por ausencia } \\
\text { de acciones preventivas } \\
\text { por parte del profesor. }\end{array}$ & $\begin{array}{l}\text { Siento que es más fácil plagiar porque los tipos de } \\
\text { evaluación académica dados por los profesores son } \\
\text { similares. } \\
\text { Siento que hay cierto tipo de tareas que me permi- } \\
\text { ten plagiar. } \\
\text { No tengo miedo de ser atrapado por el profesor } \\
\text { cuando copio. } \\
\text { Creo que el profesor no podría identificar si yo pla- } \\
\text { giara. } \\
\text { El profesor no sanciona a los estudiantes que come- } \\
\text { ten plagio. }\end{array}$ \\
\hline
\end{tabular}

Fuente: elaboración y traducción propia con base en Smith et al. (2007) y aportaciones de Hosny y Shameem (2014), Cebrián-Robles et al. (2018), López (2014) y Schrimsher et al. (2011).

\section{Fiabilidad y validez}

Al ser una traducción y adaptación propia, se requiere confirmar la fiabilidad y validez de los constructos (tablas 3, 4 y 5). Lloret, Ferreres, Hernández y Tomás (2014) presentan una interesante discusión sobre la pertinencia de los métodos actualizados para validación psicométrica de un instrumento de medición, y recomiendan emplear tanto el Análisis Factorial Exploratorio (AFE) para identificar el número y composición de los factores, como el Análisis Factorial Confirmatorio (AFC) para resumir las puntaciones observadas en un conjunto grande de variables. Es 
necesario señalar que Smith et al. (2007) construyeron la escala únicamente empleando el AFE y usando la extracción de componentes principales con rotación varimax, cuando Llorent et al. (2014) sugieren el empleo de la rotación oblimin con una extracción de máxima verosimilitud.

En relación con la homogeneidad y consistencia interna de las medidas, se calcularon los valores del Alfa de Cronbach, obteniendo valores aceptables por encima de .70 (APHP: $a=.78$, CPEP: $a=.75$ y FPPP: $a=.70$ ) (Milton, 2010).

Para la realización del AFE se verificaron los supuestos para este análisis multivariante mediante las pruebas de Kaiser-Meyer-Olkin (KMO), obteniendo valores por encima de .5, y las pruebas de esfericidad de Bartlett con valores de $p<.001$, lo que permite proceder con el análisis factorial exploratorio (Pérez-Gil, Chacón, Moreno, 2000).

El AFE se realizó mediante el método de extracción de factores por máxima verosimilitud con rotación oblimin directo (Lloret-Segura et al., 2014). En los resultados del AFE se observan cargas factoriales superiores a .30, cumpliendose así los criterios para mantener todos los ítems (Williams, Onsman, Brown, 2010). Las varianzas explicadas muestran porcentajes aceptables por encima de 35\%; sin embargo, algunas de las comunalidades presentaron valores relativamente bajos.

Para validar el modelo teórico basado en los resultados del AFE, y en virtud del resultado de algunos ítems en las comunalidades, se realizó un Análisis Factorial Confirmatorio (AFC) para demostrar la validez de la estructura factorial previamente obtenida, así como la validez de las deducciones teóricas inferidas en él (Pérez-Gil et al., 2000).

El estadístico $x^{2}$ es el tradicional índice de ajuste en el AFC, en donde valores de probabilidad mayores a .05 implican que el modelo teórico se ajusta a los datos empíricos (Valdés-Cuervo, García-Vázquez, Torres-Acuña, Urías-Murrieta, Grijalva-Quiñonez, 2019). Los valores obtenidos en los tres modelos permiten corroborar la estructura propuesta. En relación con los índices de ajuste, se presenta la raíz media cuadrada de los residuos (SRMR), el índice ajustado de bondad de ajuste (AGFI) y el índice de aproximación de la raíz de cuadrados medios del error (RMSEA), los cuales muestran valores satisfactorios. Los índices de ajuste de incremento, índice de ajuste no normado (TLI) y el índice de ajuste comparativo (CFI) obtuvieron también valores aceptables, de acuerdo con lo descrito por diversos autores (Manzano, Zamora, 2010; Valdés-Cuervo et al., 2019). 
Tabla 3. Media, desviación estándar, carga factorial y comunalidades para la variable actitudes personales hacia el plagio (APHP)

\begin{tabular}{|c|c|c|c|c|}
\hline Ítem & M & $D E$ & $\begin{array}{c}\text { Carga } \\
\text { factorial }\end{array}$ & $h_{2}$ \\
\hline No tengo ganas de trabajar ni aprender a evitar el plagio. & 2.12 & 1.21 & .81 & .66 \\
\hline $\begin{array}{l}\text { No veo la necesidad de tener conocimiento sobre el plagio } \\
\text { para mi futuro. }\end{array}$ & 2.07 & 1.23 & .68 & .46 \\
\hline $\begin{array}{l}\text { Quiero evitar el trabajo duro y por eso mejor copio de in- } \\
\text { ternet. }\end{array}$ & 2.28 & 1.19 & .59 & .35 \\
\hline No me interesa el tema de plagio. & 2.23 & 1.14 & .62 & .38 \\
\hline Cometo plagio porque todos lo hacen. & 2.16 & 1.17 & .54 & .30 \\
\hline
\end{tabular}

Nota: Fiabilidad $\alpha=.78$. Análisis Factorial Exploratorio: Método de Extracción de máxima verosimilitud, con rotación oblimin directo, $\mathrm{KMO}=.77\left(\chi^{2}=276.74 ; \mathrm{gl}=10 \mathrm{p}=.000\right)$ con una varianza total explicada de 43.56\%, $\mathrm{h}^{2}$ comunalidad. Análisis Factorial Confirmatorio: $\chi^{2}=18.87, \mathrm{gl}=5, \mathrm{p}=.002$, $\mathrm{SRMR}=.05, \mathrm{AGFI}=.92, \mathrm{TLI}=.94, \mathrm{CFI}=.97$, RMSEA $=.08 \mathrm{IC} 90[.02-.15]$.

En la tabla 4 se presentan los resultados del AFE y AFC para la variable condiciones para evitar el plagio, cuya estructura es de dos factores: conciencia y competencias para evitar el plagio.

Tabla 4. Media, desviación estándar, carga factorial y comunalidades para los factores de la variable. Condiciones para Evitar el Plagio (CPEP)

\begin{tabular}{|c|c|c|c|c|c|}
\hline \multirow{2}{*}{ Ítem } & \multirow{2}{*}{$M$} & \multirow{2}{*}{$D E$} & \multicolumn{2}{|c|}{ Carga factorial } & \multirow{2}{*}{$h^{2}$} \\
\hline & & & Factor 1 & Factor 2 & \\
\hline No entiendo lo que es plagio. & 1.88 & 1.28 & .30 & & .23 \\
\hline No veo el plagio como un problema. & 2.46 & 1.25 & .97 & & .86 \\
\hline Siento que no es importante reconocer al escritor original. & 2.26 & 1.41 & .30 & & .15 \\
\hline No sé citar correctamente. & 2.62 & 1.27 & .80 & & .49 \\
\hline No tengo la confianza parar preparar una buena tarea. & 2.23 & 1.13 & & .37 & .12 \\
\hline Me resulta difícil construir oraciones. & 2.33 & 1.26 & & .57 & .41 \\
\hline Tengo pocas habilidades de investigación. & 2.51 & 1.26 & & .52 & .29 \\
\hline Tengo dificultad para entender artículos en inglés. & 3.09 & 1.43 & & .60 & .42 \\
\hline $\begin{array}{l}\text { No sé cómo reconocer adecuadamente el autor a través } \\
\text { de citas. }\end{array}$ & 2.39 & 1.23 & & .68 & .45 \\
\hline
\end{tabular}

Nota: Fiabilidad: $\alpha=.75$. Análisis Factorial Exploratorio: Método de Extracción de máxima verosimilitud, con rotación oblimin directo, $\mathrm{KMO}=.78\left(\chi_{2}=384.76 ; \mathrm{gl}=36 \mathrm{p}=.000\right)$ con una varianza explicada de $38.5 \%$. h2 comunalidad, Factor $1=$ Conciencia para evitar el plagio, Factor $2=$ Competencias para evitar el plagio. Análisis Factorial Confirmatorio: $\chi 2=41.83, \mathrm{gl}=25, \mathrm{p}=.019, \mathrm{SRMR}=.08, \mathrm{AGFI}=.92, \mathrm{TLI}=.93, \mathrm{CFI}=.95, \mathrm{RMSEA}=.05 \mathrm{IC} 90[.02-.08]$. 
La tabla 5 presenta los resultados del AFE y AFC para la variable facilidad para plagiar, que se define operacionalmente como la percepción que tiene el estudiante sobre las facilidades que tiene para realizar plagio, principalmente por la falta de acciones para evitarlo por parte de los profesores.

Tabla 5. Media, desviación estándar, carga factorial y comunalidades para la variable Facilidad para Plagiar (FPPP)

\begin{tabular}{lcccc}
\hline \multicolumn{1}{c}{ Ítem } & $\boldsymbol{M}$ & $\boldsymbol{D} E$ & $\begin{array}{c}\text { Carga } \\
\text { factorial }\end{array}$ & $\boldsymbol{h}^{2}$ \\
\hline Siento que es más fácil plagiar porque los tipos de evaluación & 2.40 & 1.18 & .81 & .66 \\
académica dadas por los profesores son similares. & & & & \\
Siento que hay cierto tipo de tareas que me permiten plagiar. & 2.77 & 1.24 & .64 & .41 \\
No tengo miedo de ser atrapado por el profesor cuando copio. & 2.21 & 1.26 & .54 & .29 \\
Creo que el profesor no podría identificar si yo plagiara. & 2.59 & 1.19 & .42 & .18 \\
El profesor no sanciona a los estudiantes que cometen plagio. & 3.06 & 1.34 & .44 & .19 \\
\hline
\end{tabular}

Nota: Fiabilidad $\alpha=.70$. Análisis Factorial Exploratorio: Método de Extracción de máxima verosimilitud, con rotación oblimin directo, $\mathrm{KMO}=.72(\chi 2=184.38 ; \mathrm{gl}=10 \mathrm{p}=.000)$ con una varianza total explicada de $35.01 \%$, h2 comunalidad. Análisis Factorial Confirmatorio: $\chi 2=12.63, \mathrm{gl}=5, \mathrm{p}=.027, \mathrm{SRMR}=.07, \mathrm{AGFI}=.92, \mathrm{TLI}=.91, \mathrm{CFI}=.95, \mathrm{RMSEA}$ $=.08 \mathrm{IC} 90[.02-.14]$.

\section{Análisis de datos}

Los datos se analizaron en primera instancia de forma descriptiva, presentando la distribución de frecuencias por cada factor evaluado en el instrumento de medición utilizado. También se realizó una prueba t para variables independientes, para establecer si existían diferencias por género, y de ANOVA para determinar si existían diferencias por promedio. Por último, se realizó un análisis de correlación y la regresión jerárquica para establecer el factor de mayor predicción sobre la variable actitudes personales hacia el plagio.

\section{Resultados}

\section{Análisis descriptivos}

$53.5 \%$ de la población en estudio fueron mujeres, sin reporte de datos perdidos para esta variable demográfica. En su mayoría (82.5\%), los estudiantes de la muestra son solteros, y 37\% trabaja además de los estudios. La edad promedio fue de 22 años, siendo el rango de 19 a 21 años (42.5\%) el más numeroso. Respecto al promedio, la media fue de 8.51 con una desviación estándar de .48. La muestra incluyó 36\% de estudiantes de tercero a quinto semestre, $43 \%$ de sexto a octavo semestre, y $21 \%$ de noveno ciclo en adelante.

El cuestionario incluye preguntas sobre las principales fuentes de consulta para las asignaciones escolares, siendo material diverso de internet (75\%) la principal fuente de consulta, 
seguido de los libros (71\%), y solo $26 \%$ acepta realizar consulta en artículos de revistas académicas especializadas.

En lo que se refiere al proceso de dar crédito a los autores, más de la mitad (59\%) afirma que incluye la cita al autor en el texto, ya sea como pie de página (16\%) o en la lista de referencias (47\%). Finalmente, 10.5\% acepta que no reconoce la fuente de información de su tarea.

En relación con las sanciones que los estudiantes perciben deben aplicarse a esta conducta, $76 \%$ lo reduce a la reprobación de la materia, algunos más severos (13.5\%) a baja temporal por un semestre, y solo 6.5\% simpatiza con castigos drásticos como la baja definitiva.

Para finalizar este apartado descriptivo, en la tabla 6 se presentan los cuartiles de la distribución de los constructos.

Tabla 6. Distribución de frecuencias de las medidas del Cuestionario Percepción del Estudiante Universitario sobre el Plagio (PEUP)

\begin{tabular}{lccccc}
\hline \multirow{2}{*}{ Niveles } & \multirow{2}{*}{ Percentil } & APHP & COEP & CMEP & FPPP \\
\cline { 2 - 6 } & & $\%$ & $\%$ & $\%$ & $\%$ \\
\hline Muy bajo & 25 & 27.0 & 41.0 & 31.0 & 29.5 \\
\hline Bajo & 50 & 27.0 & 12.5 & 24.0 & 25.0 \\
\hline Moderado & 75 & 23.0 & 13.0 & 24.0 & 24.5 \\
\hline Alto & 100 & 23.0 & 33.5 & 21.0 & 21.0 \\
\hline
\end{tabular}

Nota: APHP: actitudes personales hacia el plagio, COEP: conciencia para evitar el plagio, CMEP: competencias para evitar el plagio, y FPPP: facilidades percibidas para el plagio.

Se aprecia que el mayor porcentaje de la población tiene conciencia de la importancia de evitar el plagio, seguido de su aceptación de la falta de competencias para evitarlo y la facilidad de condiciones que perciben tienen para realizarlo sin consecuencias en su desempeño académico.

\section{Diferencia de medias}

En primer término se presentan los resultados de la prueba t, relacionada con el género, en donde solo el factor de competencias para evitar el plagio no presentó diferencias estadísticamente significativas. Se aprecia que la media más alta en todos los factores la presentan los varones, donde el tamaño del efecto más importante es la facilidad percibida para cometer el plagio, diferencias que se explican en $40 \%$ en función del género. 
Tabla 7. Diferencias de género en relación con los factores del cuestionario Percepción del Estudiante Universitario sobre el Plagio

\begin{tabular}{lcccccccc}
\hline \multirow{2}{*}{ Factores } & \multicolumn{3}{c}{ Hombre } & \multicolumn{2}{c}{ Mujer } & \multirow{2}{*}{$\boldsymbol{t}$} & $\boldsymbol{p}$ & $\begin{array}{c}\boldsymbol{d} \text { de } \\
\text { Cohen }\end{array}$ \\
\cline { 2 - 5 } & $\boldsymbol{M}$ & $\boldsymbol{S D}$ & $\boldsymbol{M}$ & $\boldsymbol{S D}$ & & & & \\
\hline Actitudes personales hacia el plagio. & 2.32 & .88 & 2.03 & .85 & 2.31 & $.022^{*}$ & 0.33 \\
\hline Conciencia para evitar el plagio. & 2.40 & .86 & 2.15 & .84 & 2.06 & $.040^{*}$ & 0.29 \\
\hline Competencia para evitar el plagio. & 2.58 & .91 & 2.47 & .78 & .91 & .360 & 0.12 \\
\hline Facilidad percibida para plagiar. & 2.78 & .82 & 2.45 & .82 & 2.84 & $.005^{* *}$ & 0.40 \\
\hline
\end{tabular}

Nota: $\mathrm{N}=200, \mathrm{p}<0.05^{*}, \mathrm{p}<0.01^{* *}$.

Acerca del promedio, la tabla 8 presenta los datos del ANOVA en relación con los factores del cuestionario Percepción del Estudiante Universitario sobre el Plagio, y únicamente las competencias para evitar el plagio presentaron diferencias estadísticas entre los grupos, siendo la media más alta la que se reporta para los alumnos de menor promedio. No obstante, el tamaño del efecto estimado con el eta parcial cuadrado reporta un valor muy bajo, por lo que esta diferencia no puede considerarse como relevante de forma práctica.

Tabla 8. Diferencias por promedio con relación a los factores del cuestionario Percepción del Estudiante Universitario sobre el Plagio

\begin{tabular}{|c|c|c|c|c|c|c|c|c|c|}
\hline \multirow{2}{*}{ Factores } & \multicolumn{2}{|c|}{$\begin{array}{l}7.00 \mathrm{a} \\
8.00\end{array}$} & \multicolumn{2}{|c|}{8.01 a 9.00} & \multicolumn{2}{|c|}{9.01 a 10} & \multirow[t]{2}{*}{$F$} & \multirow{2}{*}{$p$} & \multirow[t]{2}{*}{$n^{2}$} \\
\hline & $M$ & $S D$ & $M$ & $S D$ & $M$ & $S D$ & & & \\
\hline $\begin{array}{l}\text { Actitudes personales } \\
\text { hacia el plagio. }\end{array}$ & 2.21 & .88 & 2.13 & .88 & 2.28 & .85 & .35 & .69 & .00 \\
\hline $\begin{array}{l}\text { Conciencia para evitar } \\
\text { el plagio. }\end{array}$ & 2.32 & .87 & 2.25 & .89 & 2.30 & .63 & .15 & .85 & .00 \\
\hline $\begin{array}{l}\text { Competencia para evitar } \\
\text { el plagio. }\end{array}$ & 2.78 & .92 & 2.47 & .84 & 2.28 & .56 & 3.62 & $.028 *$ & .04 \\
\hline $\begin{array}{l}\text { Facilidad percibida } \\
\text { para plagiar. }\end{array}$ & 2.66 & .78 & 2.60 & .88 & 2.45 & .70 & .47 & .62 & .00 \\
\hline
\end{tabular}

Nota: $\mathrm{N}=200, \mathrm{p}<0.05^{*}, \mathrm{p}<0.01^{* *}$.

\section{Análisis de correlación}

Con la finalidad de establecer primero la relación entre los factores de las tres variables, se realizó un análisis de correlación de Pearson, que demuestra que los constructos tienen una relación positiva significativa (tabla 9). Se aprecia que las actitudes personales hacia el plagio presentan el valor de correlación más elevado con respecto al factor conciencia para evitar el plagio. 
Tabla 9. Media, Desviación Estándar y valores de correlación de las medidas del cuestionario Percepción del Estudiante Universitario sobre el Plagio (PEUP)

\begin{tabular}{lcccccc}
\hline & $\mathbf{M}$ & $\mathbf{D E}$ & $\mathbf{1}$ & $\mathbf{2}$ & $\mathbf{3}$ & $\mathbf{4}$ \\
\hline 1) Actitudes personales hacia el plagio & 2.71 & .87 & - & & & \\
2) Conciencia para evitar el plagio & 2.19 & .94 & $.585^{* *}$ & - & & \\
3) Competencias para evitar el plagio & 2.52 & .85 & $.570^{* *}$ & $.391^{* *}$ & - & \\
4) Facilidad percibida para plagiar & 2.60 & .83 & $.417^{* *}$ & $.244^{* *}$ & $.32 * *$ & - \\
\hline
\end{tabular}

Nota: $* p<.05, * * p<.01, * * * p<.001$.

\section{Análisis de regresión jerárquica}

Una vez establecida la relación entre las variables, se procedió a realizar una regresión jerárquica que permitiera identificar si los factores personales del estudiante, como son: la conciencia para evitar el plagio y las competencias para evitarlo, o contextuales tales como las facilidades percibidas para realizar el plagio sin que los profesores sancionen dicha conducta, permiten predecir las actitudes personales hacia el plagio.

El primer factor, la conciencia para evitar el plagio, es el que explica la mayor parte del fenómeno $(\beta=.39, p=.000)$, seguido de su factor asociado, las competencias para evitarlo $(\beta=.33, p$ $=.000$ ) y por último, las condiciones percibidas por el estudiante que le permiten realizar plagio sin consecuencias a la evaluación de su desempeño académico $(\beta=.27, p=.000)$ (tabla 10).

Tabla 10. Resumen del análisis de regresión jerárquica para las variables conciencia para evitar el plagio, competencias para evitar el plagio y facilidad percibida para plagiar que explican las actitudes personales hacia el plagio $(\mathrm{N}=200)$

\begin{tabular}{lccccc}
\hline \multicolumn{1}{c}{ Variable } & $\mathbf{B}$ & $95 \% \mathrm{Cl}$ & $\boldsymbol{\beta}$ & $\mathrm{R} \mathbf{2}$ & $\mathbf{\Delta R \mathbf { 2 }}$ \\
\hline Paso 1: Conciencia para evitar el plagio. & .36 & {$[.26-.48]$} & $.39^{* * *}$ & $.34^{* * *}$ & $.34^{* * *}$ \\
Paso 2: Competencias para evitar el plagio. & .34 & {$[.23-.45]$} & $.33^{* * *}$ & $.48^{* * *}$ & $.13^{* * *}$ \\
Paso 3: Condiciones percibidas para el plagio. & .28 & {$[.17-.38]$} & $.27^{* * *}$ & $.53^{* * *}$ & $.06^{* * *}$ \\
\hline
\end{tabular}

Nota: $* \mathrm{p}<.05 ; * * \mathrm{p}<.01 ; * * * \mathrm{p}<.001$.

\section{Discusión}

Los resultados señalan que los factores personales tienen un mayor peso en la decisión de plagiar, seguido de la facilidad con que perciben los estudiantes de que esto puede realizarse. Se destaca que solo $10.5 \%$ de los estudiantes acepta que no le da crédito a los autores originales cuando realizan sus asignaciones académicas, pero $41 \%$ de la población encuestada acepta tener conciencia sobre la importancia que tiene conocer lo que es el plagio y cómo evitarlo. También se corrobora que internet es la principal fuente de consulta (75\% de los encuestados). Asimismo, en el tamaño del efecto que presenta el factor facilidad para plagiar, $40 \%$ de las dife- 
rencias se explican por el género, donde los varones tienen una media más alta que las mujeres; al igual que con respeco a la escala, los valores de manera genérica son bajos.

Estos resultados coinciden con diversos estudios (Culwin, Lancaster, 2001; Smith et al., 2007), pero se destaca el señalar a la conciencia del estudiante como elemento primordial que reafirma la premisa inicial de que la problemática de plagio es un tema de ética y valores, tanto individuales como colectivos.

Los resultados demuestran lo que señala Berlinck (2011) al indicar que, a pesar de que las instituciones puedan detectar el plagio, su eliminación no solo es el resultado de su detección y castigo, sino que debe ir acompañado de políticas de prevención.

Jones et al. (2006) proponen una serie de acciones que pueden ser de fácil implementación desde el aula. A manera de resumen, podemos señalar tres vías de acción: a) curriculares: la integración de políticas de integridad académica durante la orientación inicial del curso; $b$ ) pedagógicas: incluir actividades de enseñanza-aprendizaje sobre el concepto de plagio y sus implicaciones éticas, así como del aprendizaje de estilos y herramientas para citar y referenciar; $y c$ ) preventivas: estas últimas dependen de aspectos presupuestales y normativos pues requieren de la infraestructura informática para su detección, y de generar normativas para su sanción.

En cuanto a la primera directriz, en México hay mucho trabajo por delante pues los programas de pregrado incluyen de manera muy superficial asignaturas como ética y valores, pero no son considerados de manera transversal (Morató, 2012) para el resto de los programas educativos.

En relación con las acciones pedagógicas hay muchos elementos por discutir, como son la apatía e indiferencia de los profesores ante la problemática o, peor aún, su carencia de destrezas para redactar sin cometer plagio. Sin embargo, es en este campo donde existen diversas acciones que pueden prevenirlo, aunque esto suponga una demanda de conocimiento sobre el tema (Hu, Sun, 2016). Los resultados del estudio señalan, aunque en menor medida, que la percepción del estudiante sobre la facilidad con que puede cometer plagio sin que el profesor se entere o lo sancione, es un predictor de las actitudes que tenga el estudiante ante esta conducta. Berlinck (2011) sugiere promover actividades de orientación al profesorado con el fin de evitar el plagio, conocer los procedimientos y regulaciones a adoptar, sobre cómo preparar adecuadamente los cursos que se impartirán para evitar el plagio, así como la detección y las responsabilidades cuando esto ocurra.

Maurer et al. (2006) relatan que la Comunidad de Educación Superior de Reino Unido adoptó una medida de asesoramiento sobre plagio mediante la detección en línea. Un programa similar tuvo México hasta enero de 2019, a través del Consorcio Nacional de Recursos de Información Científica y Tecnológica, operado por el Consejo Nacional de Ciencia y Tecnología, donde con el programa Turnitin e iThenticate ofrecía la oportunidad de verificar el nivel de 
plagio de los trabajos de investigadores y estudiantes de posgrado, pero lamentablemente, por motivos presupuestales, se dejó de brindar este servicio. No se dispone hasta el momento de estadísticas que permitan valorar los beneficios que ofreció este servicio, pero sin duda, de haber continuado el apoyo, se hubiera podido medir el alcance de esta estrategia.

Por último, la directriz institucional debe centrarse en la prevención, pero también en su definición operacional y sanciones. Vera (2016) subraya que las organizaciones educativas tienen la autonomía relativa para establecer sus propias reglas de funcionamiento $y$, por ende, establecer sus propias sanciones. Ante esta premisa, lo esencial que los códigos de ética institucional deben contener es una definición clara y operativa de lo que abarca el plagio, así como las diferentes sanciones que deben ser implementadas por profesores y autoridades académicas, las cuales deben ir acompañadas de mecanismos de seguimiento, ya sea de pares académicos o de comisiones responsables de dar certeza a los procedimientos establecidos.

El estudio presenta como limitante el alcance transeccional del mismo, y que solo incluyó un tipo de disciplina en particular, lo que deja abierta la posibilidad de réplica en poblaciones heterogéneas que permitan un comparativo por área del conocimiento. Sin embargo, además de los resultados, aporta la adaptación y validación de un instrumento en idioma español que permite evaluar la actitud del estudiante frente al plagio. Si bien el cuestionario es perfectible, presenta una primera versión del instrumento en el idioma español para estudiantes de pregrado.

\section{Referencias}

Bautista, F.; P. Sánchez-Escobedo; P. Canto-Herrera (2017). Plagio en los posgrados de Ciencias Sociales. Educación y Ciencia, 6(47), 82-97. http://educacionyciencia.org/index.php/educacionyciencia/article/view/406

Berlinck, R. G. S. (2011). The Academic Plagiarism and its Punishments - A Review. Brazilian Journal of Pharmacognosy, 21(3), 365-372. https://doi.org/10.1590/S0102-695X2011005000099

Bhutto, F.; K. F. Mamdani (2019). A Correlational Study between the Big Five Personality Traits and Plagiarism. International Journal of Humanities and Cultural Studies, 6(1), 103-115. https:// www.ijhcs.com/index.php/ijhcs/article/view/3320/3094

Cardoso, R. M. (2016). Plágio acadêmico: a responsabilidade das associações científicas. Intercom: Revista Brasileira de Ciências Da Comunicação, 39(3), 187-200. https://doi.org/10.1590/1809$\underline{58442016311}$

Cebrián-Robles, V.; M. Raposo-Rivas; M. Cebrián-de-la-Serna; J. A. Sarmiento-Campos (2018). Perception of Academic Plagiarism by Spanish University Students. Educación XX1, 21(2), 105-129. https://doi.org/10.5944/educXX1.20062 
Chu, S. K. W.; X. Hu; J. Ng (2019). Exploring Secondary School Students' Self-perception and Actual Understanding of Plagiarism. Journal of Librarianship and Information Science. https:// doi.org/10.1177/0961000619872527

Comas, R.; J. Sureda; M. Trobat (2011). Prácticas de citación y plagio académico en la elaboración textual del alumnado universitario. Teoría de la Educación. Educación y Cultura en la Sociedad de la Información, 12(1), 359-385. http://www.redalyc.org/articulo.oa?id=201021400017

Culwin, F.; T. Lancaster (2001). Plagiarism Issues for Higher Education. Vine, 31(2), 36-41. https:// doi.org/10.1108/03055720010804005

Díaz, D.; J. Brito; V. Nieto; W. Muñoz (2019). Efectos de la retroalimentación sobre la disminución del plagio académico en estudiantes de bachillerato. Revista Innova Educación, 1(4), 468489.https://doi.org/10.35622/j.rie.2019.04.005

Enders, W.; G. Hoover (2006). Plagiarism in the Economics Profession: A Survey. Challenge, 49(5), 92-107. https://doi.org/10.2753/0577-5132490506

Gantús, F., (2016). Conocimientos colectivos, obras particulares. Perfiles Educativos, 38(154), 12-19. http://www.scielo.org.mx/scielo.php?script=sci arttext\&pid=S0185-26982016000400017

Gómez-Espinosa, M.; V. Francisco; P. Moreno-Ger (2016). The Impact of Activity Design in Internet Plagiarism in Higher Education. Comunicar, 24(48), 39-47. https://doi.org/10.3916/C48-2016-04

Hannabuss, S. (2001). Contested Texts: Issues of Plagiarism. Library Management, 22(6/7), 311318. https://doi.org/10.1108/EUM0000000005595

Hernández-Islas, M. (2016). El plagio académico en la investigación científica. Consideraciones desde la óptica del investigador de alto nivel. Perfiles Educativos, 38(153), 120-135. https:// doi.org/10.22201/iisue.24486167e.2016.153.57639

Hosny, M.; F. Shameem (2014). Attitude of Students Towards Cheating and Plagariasm: University Case Study. Journal of Applied Sciences, 14(8), 748-757. http://docsdrive.com/pdfs/ansinet/jas/2014/748-757.pdf

Hu, G.; X. Sun (2016). Chinese University EFL Teachers' Knowledge of and Stance on Plagiarism. Comunicar, 24(48), 29-37. https://doi.org/10.3916/C48-2016-03

Inclán, C. (2016). Ctrl-C, Ctrl-V. Perfiles Educativos, 38(154), 6-11. http://www.scielo.org.mx/pdf/peredu/v38n154/0185-2698-peredu-38-154-00006.pdf

Jones, D. L. R. (2011). Academic Dishonesty: Are More Students Cheating? Business Communication Quarterly, 74(2), 141-150. https://doi.org/10.1177/1080569911404059

Jones, K. O.; J. Reid; R. Bartlett (2006). E-Learning and E-Cheating. 3rd E-Learning Conference, 45-48. Coimbra, Portugal.

Kattan, A. E.; F. Alshomer; A. K. Alhujayri; F. Alfaqeeh; Y. Alaska; K. Alshakra (2017). The Practice and Attitude towards Plagiarism among Postgraduate Trainees in Saudi Arabia. Journal of Health Specialties, 5(4), 181-184. https://doi.org/10.4103/jhs.JHS 
Lloret-Segura, S.; A. Ferreres-Traver; A. Hernández-Baeza; I. Tomás-Marco (2014). El análisis factorial exploratorio de los ítems: una guía práctica, revisada y actualizada. Anales de Psicología, 30(3), 1151-1169. https://doi.org/10.6018/analesps.30.3.199361

López, J. (2014). Analyzing and Reducing Plagiarism at University. European Journal of Education and Psychology, 7(2), 131-140. https://doi.org/10.1989/ejep.v7i2.186

Manzano, A.; S. Zamora (2010). Sistema de ecuaciones estructurales: 4. México: Centro Nacional de Evaluación para la Educación Superior, A.C.

Maurer, H., F. Kappe; B. Zaka (2006). Plagiarism - A Survey. Journal of Universal Computer Science, 12(8), 1050-1084. https://doi.org/10.3217/jucs-012-08-1050

Merino Soto, C; R. Charter (2010). Modificación Horst al Coeficiente KR - 20 por Dispersión de la Dificultad de los Ítems. Interamerican Journal of Psychology, 44(2), 274-278.

Meuschke, N.; B. Gipp (2013). State-of-the-Art in Detecting Academic Plagiarism. International Journal for Educational Integrity, 9(1), 50-71. https://doi.org/https://doi.org/10.21913/JJEl.v9i1.847

Milton, V. (2010). Confiabilidad y coeficiente Alpha de Cronbach Licenciado Educación Mención Matemática y Física. Profesor de Educación Integral, 12(2), 248-252.

http://redalyc.uaemex.mx/src/inicio/ArtPdfRed.jsp?iCve=99315569010

Miranda, A. (2013). Plagio y ética de la investigación científica. Revista Chilena de Derecho, 40(2), 711-726. https://doi.org/10.4067/s0718-34372013000200016

Morales, M. A. (2016). El plagio académico desde la perspectiva del derecho de autor. Revista Espacio I+D Innovación Más Desarrollo, 5(11), 104-120.

https://doi.org/10.31644/imasd.11.2016.a06

Morató, Y. (2012). Una reflexión necesaria sobre el plagio en el EEES. Revista UPO Innova, 1, $361-$ 368. https://www.upo.es/revistas/index.php/upoinnova/article/view/113

Pérez-Gil, J. A.; S. Chacón; R. Moreno (2000). Validez de constructo: El uso de análisis factorial exploratorio-confirmatorio para obtener evidencias de validez. Psicothema, 12(Supl. 2), 442-446. http://www.psicothema.es/pdf/601.pdf

Rohwer, A.; E. Wager; T. Young; P. Garner (2018). Plagiarism in Research: A Survey of African Medical Journals. BMJ Open, 8(11), 1-8. https://doi.org/10.1136/bmjopen-2018-024777

Ruipérez, G.; J. C. García-Cabrero (2016). Plagiarism and Academic Integrity in Science. Comunicar. Media Education Research Journal, 24(48), 9-17. http://dx.doi.org/10.3916/C48-2016-01

Schrimsher, R. H.; L. A. Northrup; S. P. Alverson (2011). A Survey of Samford University Students Regarding Plagiarism and Academic Misconduct. International Journal for Educational Integrity, 7(1), 3-17. https://doi.org/10.21913/IJEl.v7i1.740

Smith, M.; N. Ghazali; S. F. Noor-Minhad (2007). Attitudes towards Plagiarism among Undergraduate Accounting Students: Malaysian Evidence. Asian Review of Accounting, 15(2), 122-146. https://doi.org/10.1108/13217340710823350 
Soto, A. (2012). El plagio y su impacto a nivel académico y profesional. E-Ciencias de la Información, 2(1), 1-13. https://doi.org/10.15517/eci.v2i1.1213

Timal, S.; F. Sánchez (2017). El plagio en el contexto del derecho de autor. Tla-Melaua. Revista de Ciencias Sociales, 11(42), 48-66. http://www.scielo.org.mx/scielo.php?script=sci arttex$\underline{\mathrm{t} \& \mathrm{pid}=\mathrm{S} 1870-69162017000200048}$

Universidad Juárez Autónoma de Tabasco (2019). Sistema de indicadores de servicios escolares para pregrado [base de datos].

Valdés-Cuervo, A. A.; F. García-Vázquez; G. M. Torres-Acuña; M. Urías-Murrieta; C. S. Grijalva-Quiñonez (2019). Medición en investigación educativa con apoyo del SPSS y el AMOS. México: Instituto Tecnológico de Sonora.

Vera, H. (2016). El plagio y la autonomía de las instituciones académicas. Perfiles Educativos, 38(154), 28-35. http://www.scielo.org.mx/pdf/peredu/v38n154/0185-2698-peredu-38-154-00028.pdf

Williams, B.; A. Onsman; T. Brown (2010). Exploratory Factor Analysis: A Five-step Guide for Novices. Journal of Emergency Primary Health Care, 8(3), 1-13. https://ajp.paramedics.org/index. php/ajp/article/view/93/90

Yankelevich, J. (2016). Mapas prestados para entender el plagio académico. Perfiles Educativos, 38(154), 20-27.

http://www.scielo.org.mx/pdf/peredu/v38n154/0185-2698-peredu-38-154-00020.pdf

Zimerman, M. (2012). Plagiarism and International Students in Academic Libraries. New Library World, 113(5-6), 290-299. https://doi.org/10.1108/03074801211226373 